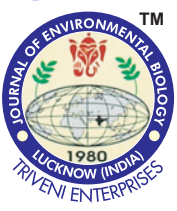

\title{
Seasonal variations in the proximate composition of Pyropia yezoensis harvested along the western and southern coast of Korea
}

\section{Authors Info}

S.M. Jung, S.G. Kang, J.H. Jeon, J.S. Son, H.J. Lee, T.H. Park, S.H. Han, W.B. Jeon and H.W. Shin*

Department of Life Science and Biotechnology, Soonchunhyang University, Asan City, Choongnam Do, 336-745, South Korea

*Corresponding Author Email : hwshin@sch.ac.kr

\section{Edited by}

Professor Chae Woo Ma

Reviewed by

Dr. Chiyoung Lim

Professor Dong Soo Hwang

\section{Abstract}

Aim: Pyropia yezoensis is a widely consumed edible seaweed that contains substantial carbohydrates, proteins, lipids, amino acids and minerals. In this study, the proximate composition, amino acid content and mineral content of $P$. yezoensis were compared at two culture sites in South Korea (Goheung and Gunsan) during same time period.

Methodology: This study followed AOAC methods for proximate composition. Amino acid and mineral were analyzed by amino acid analyzer and inductively coupled plasma.

Results: The highest measured protein contents were $39.66 \pm 0.49 \%$ in December at Goheung and $38.77 \pm 0.59 \%$ in January at Gunsan. Proximate compositions did not differ significantly between Gunsan and Goheung. Among amino acids, isoleucine, leucine, threonine, lysine, histidine and valine were present at higher concentrations during November and December harvesting periods. The levels of $\mathrm{Ca}$ and $\mathrm{P}$ were higher in Pyropia at Gunsan from November to February compared with Pyropia at Goheung.

Interpretation: Clear temporal and spatial variations were observed in amino acid and mineral contents, but not in proximate composition. Our study provides important information about the nutritive properties of $P$. yezoensis.

Key words: Amino acids, Carbohydrate, Pyropia yezoensis, Seasonal variations

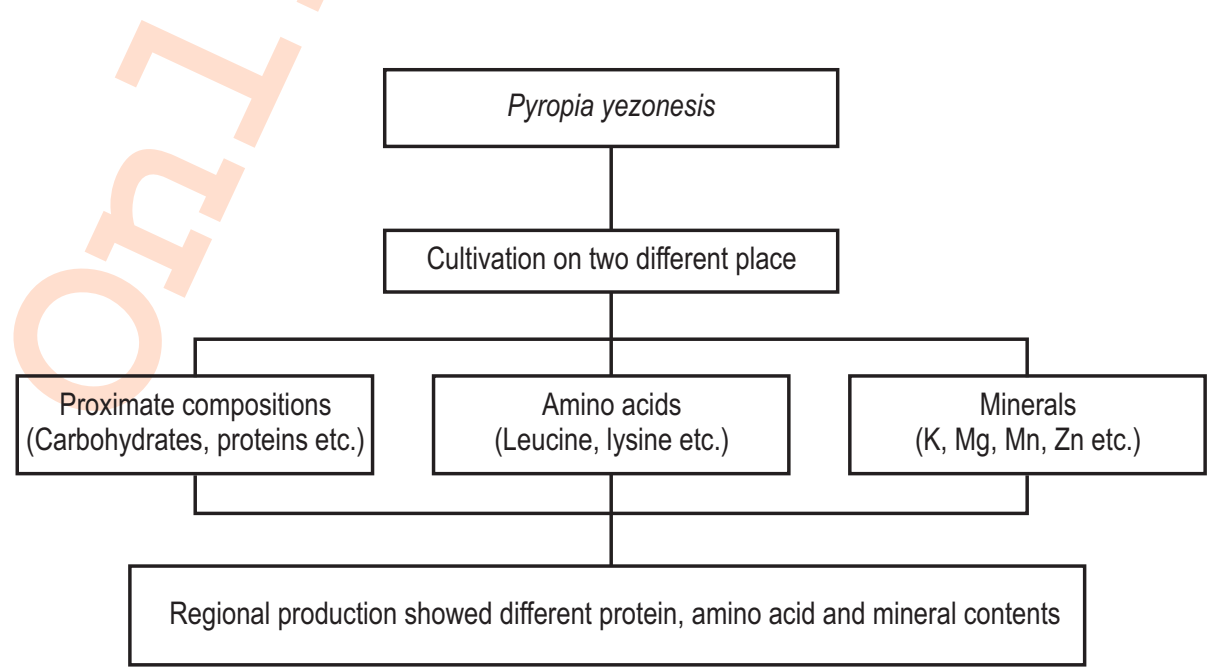

How to cite : Jung, S.M., S.G. Kang, J.H. Jeon, J.S. Son, H.J. Lee, T.H. Park, S.H. Han, W.B. Jeon and H.W. Shin: Seasonal variations in the proximate composition of Pyropia yezoensis harvested along the western and southern coast of Korea. J. Environ. Biol, 40, 932-939 (2019). DOI : http://doi.org/10.22438/jeb/40/5(SI)/SI-16 


\section{Introduction}

In many countries, marine algae have significant nutritional and environmental resources. Edible algae are widely consumed in Asian countries in fresh and dried forms and are often used as ingredients in certain dishes. In particularly, the red alga genus Pyropiais commonly used for food and is the basis of large industries in Korea, Japan and China (Nisizawa et al., 1987). Although algae are not widely consumed in Europe compared with Asia, they have gained popularity as a natural source of bioactive compounds. Owing to its nutritive value and health benefits, Pyropia consumption has increased in Western countries in recent years (Dawczynski et al., 2007). Because of its low fat content and proteins and carbohydrates that cannot be entirely digested by human intestinal enzymes, it contributes relatively few calories to the diet (Jurkovic et al., 1995; Kishi et al., 1982; Lahaye and Kaeffer, 1997).

Dried algae contain large quantities of carbohydrates, proteins, ash, lipids, minerals and other constituents. For example, the carbohydrate and protein levels of Pyropia are 34.3-50.2\% and 25-50\% (Noda, 1993; Jung et al., 2016a). Shin et al. (2013) reported carbohydrate, protein and lipid contents of $39.47-47.2 \%, 29.8-40.8 \%$ and $0.7-1.1 \%$, in algae harvested from the Seochun coast of Korea. On the Wando coast of Korea, the protein contents of $P$. tenera and $P$. haitanesis ranged from $32.16 \%$ to $36.88 \%$, respectively (Hwang et al., 2013).

Pyropia contain high levels of essential amino acids such as leucine, lysine, threonine, valine, phenyl alanine, tyrosine, methionine and cysteine. Cian et al. (2013) analyzed the amino acid composition of Pyropia and reported high levels of leucine $\left(7.38 \mathrm{~g}^{100 \mathrm{ml}^{-1}}\right)$, lysine $\left(6.01 \mathrm{~g} 100 \mathrm{ml}^{-1}\right)$, threonine $\left(5.91 \mathrm{~g}^{100 \mathrm{ml}^{-1}}\right)$ and valine $\left(5.85 \mathrm{~g}^{100 \mathrm{ml}^{-1}}\right)$. Hwang et al. (2013) found that

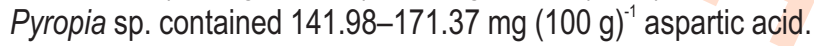
Amino acids such as alanine, aspartic acid and glycine are responsible for peculiar flavors of Pyropia sp. (Shin, 1997; Mabeau et al., 1992).

Pyropia may be an important source of certain minerals that are not abundant in terrestrial plants (Ortega-Calvo, 1993). Ruperez (2002) identified various minerals by atomic absorption spectrophotometry, which revealed high levels of macrominerals

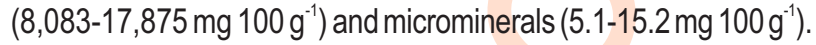
Similarly, $P$. purpurea was found to contain high levels of

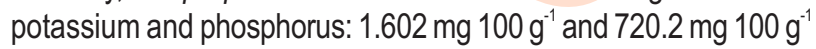
(Toboada et al., 2013). Other recent studies have reported iron levels of $21.3 \mathrm{mg}(100 \mathrm{~g})^{-1}$ and $10.3 \mathrm{mg}(100 \mathrm{~g})^{-1}$ in $P$. columbina and $P$. tenera, respectively (Ruperez, 2002; Perez et al., 2007). However, the nutritional value of algae may be affected by environmental factors such as salinity, water and air temperatures, and $\mathrm{pH}$, as well as by seasonal and spatial variations (Shin et al., 2013; Phillips, 1990). Most environmental parameters vary according to season, and changes in ecological conditions can stimulate or inhibit biosynthesis of several nutrients (Lobban et al., 1985). However, the associations between spatial and temporal variables and the chemical composition and properties of Pyropia have not yet been investigated; most studies to date have focused on other seaweed species.

In the present study, Pyropia yezoensis was cultivated at Gunsan on the western coast of South Korea simultaneously with $P$. yezoensis cultivated at Goheung on the southern coast. Proximate compositions, amino acid and mineral contents, and environmental conditions between these two sampling sites were compared.

\section{Materials and Methods}

Samples : $P$. yezoensis samples were collected from cultivation sites at Gunsan on the western coast of South Korea, and Goheung, on the southern coast of South Korea. Sampling was carried out every month from November 2015 to March 2016. P. yezoensis samples were transported in plastic bags in an ice box at low temperature. The weight of the transported wet samples was measured, and samples were then dried in an oven at $60^{\circ} \mathrm{C}$ for $24 \mathrm{hr}$. To complete the drying process, the samples were placed in an oven three times for $1 \mathrm{hr}$ at $105^{\circ} \mathrm{C}$. All samples were ground and passed through a $0.5 \mathrm{~mm}$ mesh sieve prior to further analysis. The proximate composition, amino acid and mineral content of these samples were compared.

Analysis of proximate composition : The total proximate composition, including carbohydrate, protein, lipid and ash was determined following the Association of Official Analytical Chemists (AOAC, 1995) method.

For protein analysis, $1 \mathrm{~g}$ of sample was placed in a Kjeldahal flask with $\mathrm{CuSO}_{4} 7 \mathrm{H}_{2} \mathrm{O}: \mathrm{K}_{2} \mathrm{SO}_{4}$ in $1: 4$ ratio and then heated. Total protein content was determined by the Kjeldahal method using fixed nitrogen:protein conversion factor of 6.25 (AOAC, 1995).

For analysis of lipid, samples were dried in an oven for 24 $\mathrm{hr}$. and then $2 \mathrm{~g}$ of sample was filtered using a micro filter tube (pore size $=8 \mu \mathrm{m}, \mathrm{ID}=25 \mathrm{~mm}, \mathrm{OD}=28 \mathrm{~mm}, \mathrm{~L}=100 \mathrm{~mm}$ ). The micro filter tube was blocked using cotton and then placed into a Soxhlet extractor. Diethyl ether $(300 \mathrm{ml})$ within a round flask was affixed to the Soxhlet siphon, which was then installed in a water bath at temperature increasing from 40 to $55^{\circ} \mathrm{C}$ for over $10 \mathrm{hr}$. The extracted sample was evaporated, after which it was dried in an oven for $30 \mathrm{~min}$ and placed in a desiccator for $1 \mathrm{hr}$ at room temperature; this process was repeated three times. Sample weight was measured at $20-25^{\circ} \mathrm{C}$. Lipid concentrations were calculated by the formula: lipids $(\%)=[\mathrm{X} /$ sample weight $] \times 100$; where, $X$ = initial weight of the round flask - final weight of the round flask (AOAC, 1995). 
For ash analysis, the ground dried samples were turned into ash by heating for $5 \mathrm{hr}$ in an electric oven at $500^{\circ} \mathrm{C}$ (AOAC, 1995).Carbohydrates were quantified as the difference of sum of protein, lipid and ash contents, subtracted from 100\% (AOAC, 1995).

Amino acids were analyzed using an amino acid analyzer (L8900, Hitachi High-Technologies, Tokyo, Japan). The sample (1 g) was hydrolyzed with $6 \mathrm{~N} \mathrm{HCl}(20 \mathrm{ml})$ and then incubated for over $20 \mathrm{hr}$ at $120^{\circ} \mathrm{C}$. The hydrolyzed sample was filtered (pore size $2.5 \mu \mathrm{m}$ ) and then evaporated using a pressure evaporator to remove acids. The sample was mixed with $10 \mathrm{ml}$ of $0.2 \mathrm{M}$ sodium citrate buffer ( $\mathrm{pH}$ 2.2), filtered using a syringe filter (pore size 0.2 $\mu \mathrm{m})$ and diluted 40 -fold with distilled water. Standard amino acid mixture solution was purchased from Wako Co. (Japan) as a reference material. The conditions and solvent system used for the amino acid analyzer are provided in Table 1.

Pulverized Pyropia $(0.5 \mathrm{~g}$ ) was placed in a beaker and carefully mixed with $1 \mathrm{ml} \mathrm{HNO}_{3}$. The mixture was placed on a hot plate at $50^{\circ} \mathrm{C}$ to digest the sample under a fume hood. After acid digestion, the contents were left to cool, and the acid was evaporated, for $30 \mathrm{~min}$. After evaporation of acid, the digested samples were transferred into a $50 \mathrm{ml}$ volumetric flask with deionized water. $\mathrm{Ca}, \mathrm{K}, \mathrm{Mg}, \mathrm{Na}, \mathrm{P}, \mathrm{Mn}, \mathrm{Fe}, \mathrm{Cu}$ and $\mathrm{Zn}$ were analyzed using an inductively coupled plasma optical emission spectrometer (Vista-PRO, Agilent Technologies, Waldbronn, Germany). Triplicate measurements were performed for each element. The concentration of each element was determined based on its standard calibration curve.

Statistical analyses : All data are presented as mean \pm SD. Student's t-tests were used to assess difference between paired means derived from the samples collected from Gunsan and Goheung. The statistical significance of differences between means (threshold: $P<0.05$ ) was estimated based on one-way ANOVAand Tukey's HSD post hoc testusing SPSS ver. 17.

\section{Results and Discussion}

South Korea is surrounded by the East, West and South Seas, with a coastline of approximately $2,413 \mathrm{~km}$. Approximately, $90 \%$ of cultivated seaweed was farmed on the West and South coasts. Cultivated species include Cauleerpa sp., Laminaria sp., Hizikia fusiformis, Monostroma sp., Codium sp. and Pyropia sp. The red alga Pyropia sp. accounts for approximately $90 \%$ of all cultivated seaweed on both coasts, including the sites of Gunsan on the west coast and Goheung on the south coast (Kim et al., 2014).

Air, water temperature and salinity were measured at the cultivation sites at Gunsan and Goheung from November 2015 to March 2016. The water temperature at Gunsan decreased from $16.38^{\circ} \mathrm{C}$ in November to $1.8^{\circ} \mathrm{C}$ in mid-January, before increasing again to $8.7^{\circ} \mathrm{C}$ in March. The water temperature at Goheung followed a similar pattern; however, it was $1-3^{\circ} \mathrm{C}$ higher than that at Gunsan on average, with greater daily fluctuations. The salinity ranged from 29.8 psu to 32.7 psu at Goheung and from $27.1 \mathrm{psu}$ to 30.4 psu at Goheung (Fig. 1). Another red algae, Gracilaria can tolerate a wide range of salinities, from about 10-40 psu, though they grow best between 25-33 psu (Gorman et al., 2017).

The proximate composition, including protein, lipid, carbohydrate and ash contents, of $P$. yezoensis were compared between Gunsan and Goheung (Table 2). Crude protein and ash contents showed statistically significant variations over time $(P<0.05)$, while crude lipid and carbohydrate contents did not vary significantly. Red seaweed contain high protein levels (GallandIrmouli et al., 1999). Pyropia spp. contained protein levels comparable to those of terrestrial plant foods, such as white soybeans (33.8\%) (Norziah and Ching, 2000).

The highest protein content was $39.66 \%$ in the samples collected at Goheung (December) and $38.77 \%$ in the Goheung samples (January). From November to February, the ash content of the Goheung samples remained fairly consistent, ranging from $5.29 \%$ to $5.55 \%$. The lowest ash content, $4.48 \%$, was observed in March. The proximate composition did not differ significantly between the Gunsan and Goheung samples. Proximate compositions may vary depending on climate, temperature, $\mathrm{pH}$, geographical differences, species and season (Fleurence 1999; Ortriz et al. 2006). Noda et al. (1971) reported that proximate composition in red algae followed temporal and spatial patterns. Conversely, the carbohydrate content was higher during the later cultivation period (Park et al. 2001). Marinho-Soriano et al. (2006) reported that nitrogen content in red algae was positively correlated with protein content but negatively with carbohydrate content, salinity and water temperature.

$P$. yezoensis harvested from Gunsan and Goheung was analyzed for essential amino acids, such as isoleucine, leucine, threonine, methionine, phenylalanine, lysine, histidine, valine and arginine. Among these amino acids, isoleucine, leucine, threonine, lysine, histidine and valine were present at higher concentrations in samples harvested in November and December. The most abundant amino acid was leucine, ranging

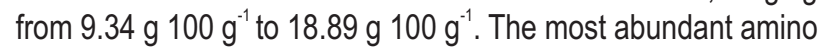
acids were threonine, phenylalanine, lysine, valine and arginine,

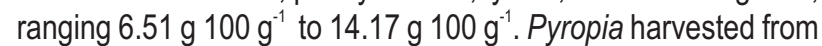
Goheung contained significantly higher concentrations of isoleucine, leucine, threonine, lysine, histidine, valine and arginine compared with Pyropia harvested from Gunsan $(P<0.05)$. Conversely, from November to January, Pyropia from Gunsan contained higher concentrations of methionine and phenylalanine compared with Pyropia from Goheung $(P<0.05)$ (Table 3). Aspartic and glutamic acids constitute a large proportion of amino acid fraction, representing $22-44 \%$ of total amino acids (Munda, 1977). Alanine, glutamic acid and aspartic acids were the most abundant amino acids (Lee et al., 2012; Jung etal., 2016b) 

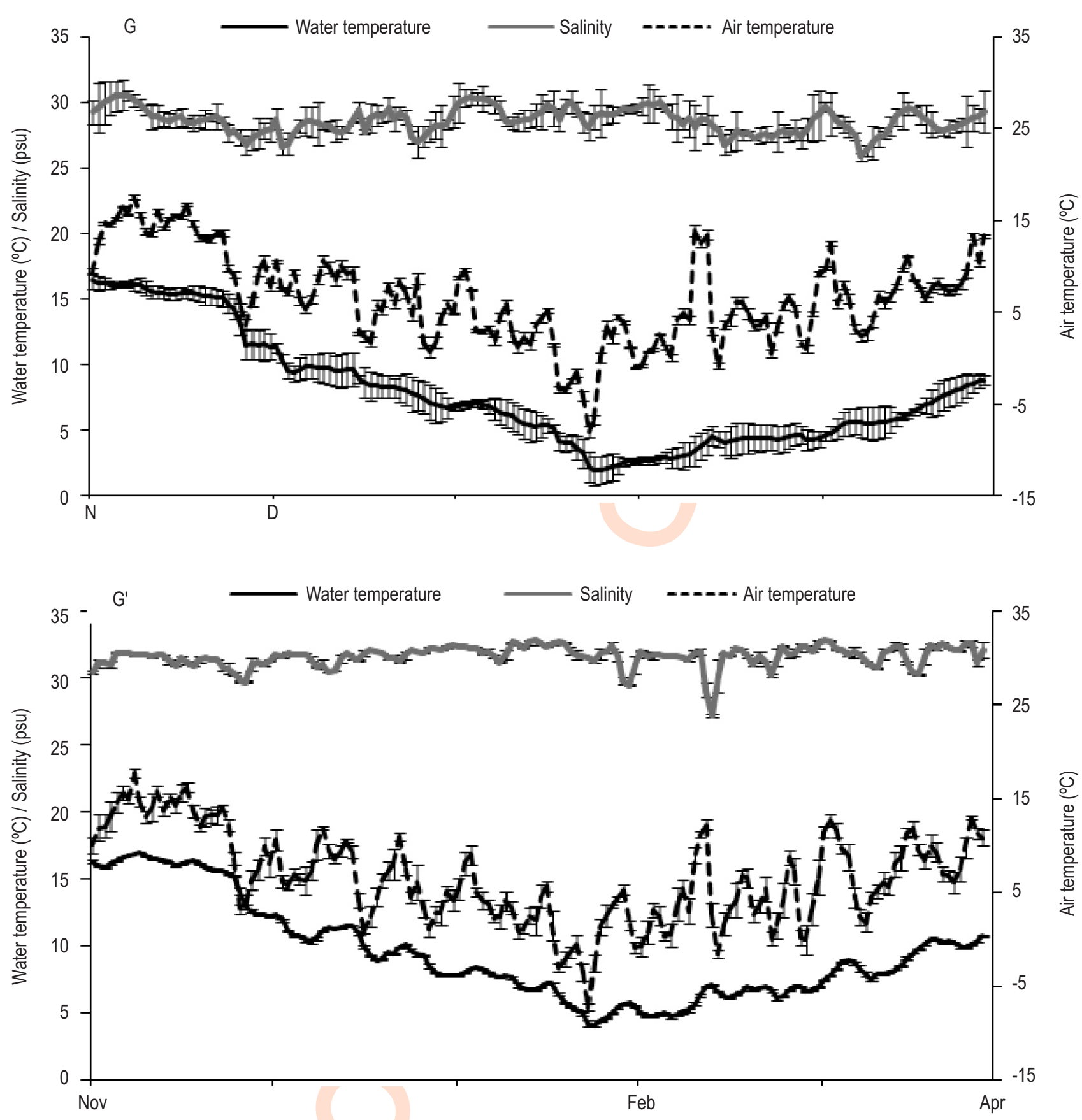

Fig. 1 : Physiological parameter of water, air temperature and salinity at Gunsan $(G)$ and Goheung $\left(G^{\prime}\right)$.

Pyropia samples contained not only essential amino acids but also non-essential amino acids, such as aspartic acid, glycine, tyrosine, serine, glutamic acid, alanine and proline. Among these amino acids, glutamic acid, aspartic acid, glycine and alanine were present high concentrations. The highest concentrations of glutamic acid and aspartic acid was $41.48 \mathrm{~g} 100 \mathrm{~g}^{-1}$ and $37.10 \mathrm{~g}$
$100 \mathrm{~g}^{-1}$, respectively $(\mathrm{P}<0.05)$, in Pyropia harvested from Gunsan in November and December. Glycine and serine were present at higher concentrations in samples collected from Goheung $(P<0.05)$. From November to January, Pyropia samples from Gunsan contained higher concentrations of tyrosine and alanine compared with the Goheung samples $(P<0.05)$ (Table 4). 
Table 1 : Conditions and solvent system of the amino acid analyzer

\begin{tabular}{|c|c|}
\hline Comparts & Conditions \\
\hline Column & 4.6 mm ID X60 mm L (packed with Hitachi custom ion exchange resin) \\
\hline Injection volume & $20 \mu \mathrm{l}$ \\
\hline Temperature & Oven $57^{\circ} \mathrm{C}$; Reactor $135^{\circ} \mathrm{C}$ \\
\hline Wavelength & $570 \mathrm{~nm}, 440 \mathrm{~nm}$ \\
\hline Flow rate & Pump 1: buffer : $0.4 \mathrm{ml} \mathrm{min}^{-1}$ Pump 2: buffer : $0.35 \mathrm{ml} \mathrm{min}^{-1}$ \\
\hline \multirow[t]{2}{*}{ Mobile phase } & Pump 1: Wako L-9800 : buffer solution : $\mathrm{PH}-1, \mathrm{PH}-2, \mathrm{PH}-3, \mathrm{PH}-4, \mathrm{H}_{2} \mathrm{O}, \mathrm{PH}-\mathrm{RG}$ \\
\hline & Pump 2:Ninhydrin solution, Ninhydrin buffer, $5 \%$ ethanol (Wako Co.) \\
\hline
\end{tabular}

Table 2 : Monthly crude protein, lipid, carbohydrate and ash contents of laver from Pyropia yezoensis (\% d. wt.)

\begin{tabular}{lllllll}
\hline & Area & Nov & Dec & Jan & Feb & Mar \\
\hline Protein & Goheung & $38.89 \pm 0.38^{\mathrm{c}}$ & $39.66 \pm 0.49^{\mathrm{bc}}$ & $39.10 \pm 0.32^{\mathrm{c}}$ & $37.82 \pm 0.20^{\mathrm{ab}}$ & $37.03 \pm 0.48^{\mathrm{a}}$ \\
& Gunsan & $38.58 \pm 0.51^{\mathrm{b}}$ & $38.33 \pm 0.20^{\mathrm{b}}$ & $38.77 \pm 0.59^{\mathrm{b}}$ & $38.13 \pm 0.20^{\mathrm{b}}$ & $37.16 \pm 0.45^{\mathrm{a}}$ \\
Lipid & Goheung & $4.44 \pm 2.89^{\mathrm{NS}}$ & $3.45 \pm 0.15$ & $2.94 \pm 0.08$ & $3.07 \pm 0.12$ & $3.03 \pm 0.12$ \\
& Gunsan & $3.45 \pm 1.06^{\mathrm{NS}}$ & $3.13 \pm 0.92$ & $3.02 \pm 0.14$ & $2.99 \pm 0.04$ & $3.08 \pm 0.13$ \\
Carbohydrate & Goheung & $51.22 \pm 3.29^{\mathrm{NS}}$ & $52.60 \pm 0.34$ & $52.45 \pm 0.33$ & $53.56 \pm 0.45$ & $55.10 \pm 0.37$ \\
& Gunsan & $53.56 \pm 1.53^{\mathrm{NS}}$ & $53.42 \pm 0.99$ & $52.58 \pm 0.36$ & $53.10 \pm 0.24$ & $54.32 \pm 0.44$ \\
Ash & Goheung & $5.45 \pm 0.22 \mathrm{~b}^{\mathrm{b}^{\mathrm{a}}}$ & $5.29 \pm 0.22^{\mathrm{b}}$ & $5.51 \pm 0.04^{\mathrm{b}}$ & $5.55 \pm 0.20^{\mathrm{b}}$ & $4.48 \pm 0.13^{\mathrm{a}}$ \\
& Gunsan & $4.42 \pm 0.15^{\mathrm{a}}$ & $5.12 \pm 0.09^{\mathrm{b}}$ & $5.63 \pm 0.10^{\text {cd }}$ & $5.78 \pm 0.08^{\mathrm{d}}$ & $5.45 \pm 0.05^{\mathrm{a}^{\mathrm{b}}}$ \\
\hline
\end{tabular}

Values are mean of three replication $\pm S D$; ${ }^{\text {acc }}$ Values with the same superscripts in each row are not significantly different at $p<0.05$; ${ }^{\text {Ns }}$ it is not statistically significant; ${ }^{*} p<0.05$, indicating significant difference between Gohueng and Gunsan group

Table 3 : Comparison of essential amino acid content of Pyropia yezoensis (g $100 \mathrm{~g}^{-1} \mathrm{~d}$. wt.)

\begin{tabular}{|c|c|c|c|c|c|c|}
\hline & & Nov & Dec & Jan & Feb & Mar \\
\hline \multirow[t]{2}{*}{ Isoleucine } & Goheung & $4.25 \pm 0.19^{c^{* *}}$ & $2.73 \pm 0.03^{a^{2+1+}}$ & $4.64 \pm 0.11^{c+4+}$ & $4.38 \pm 0.20^{c^{* t}}$ & $3.83 \pm 0.21^{\mathrm{b}+\mathrm{m}}$ \\
\hline & Gunsan & $3.59 \pm 0.10^{\text {cd }}$ & $2.19 \pm 0.06^{\mathrm{a}}$ & $3.80 \pm 0.07^{\mathrm{d}}$ & $3.40 \pm 0.18^{\mathrm{c}}$ & $2.47 \pm 0.01^{b}$ \\
\hline \multirow[t]{2}{*}{ Leucine } & Goheung & $18.89 \pm 0.81^{c}$ & $14.24 \pm 0.10^{\mathrm{a}^{a *}}$ & $17.03 \pm 0.49^{c^{c+}}$ & $16.42 \pm 0.79^{\mathrm{bc}}$ & $14.99 \pm 0.79^{\mathrm{ab}=}$ \\
\hline & Gunsan & $17.89 \pm 0.50^{d}$ & $12.11 \pm 0.36^{b}$ & $15.54 \pm 0.24^{c}$ & $14.84 \pm 0.84^{\circ}$ & $9.34 \pm 0.02^{\mathrm{a}}$ \\
\hline \multirow[t]{2}{*}{ Threonine } & Goheung & $11.31 \pm 0.51^{b^{*}}$ & $8.22 \pm 0.08^{\mathrm{a}^{* t}}$ & $12.39 \pm 0.33^{\mathrm{b**}}$ & $11.56 \pm 0.52^{b^{*}}$ & $11.27 \pm 0.57^{b^{* * *}}$ \\
\hline & Gunsan & $10.19 \pm 0.30^{c}$ & $7.04 \pm 0.21^{\mathrm{a}}$ & $11.23 \pm 0.17^{d}$ & $10.09 \pm 0.52^{\circ}$ & $8.16 \pm 0.02^{b}$ \\
\hline \multirow[t]{2}{*}{ Methionine } & Goheung & $1.15 \pm 0.05^{b}$ & $1.40 \pm 0.01^{\mathrm{c}}$ & $0.31 \pm 0.01^{\mathrm{a}}$ & $4.00 \pm 0.17^{e^{*}}$ & $3.67 \pm 0.18^{\mathrm{d}+t+}$ \\
\hline & Gunsan & $2.23 \pm 0.05^{\mathrm{bt+m}}$ & $2.26 \pm 0.11^{\mathrm{b}+\mathrm{m}}$ & $1.40 \pm 0.02^{\mathrm{a}^{* a+}}$ & $3.69 \pm 0.18^{d}$ & $2.81 \pm 0.08^{c}$ \\
\hline \multirow[t]{2}{*}{ Phenylalanine } & Goheung & $10.33 \pm 0.43^{\mathrm{bc}}$ & $8.77 \pm 0.07^{\mathrm{a}^{+4+}}$ & $10.81 \pm 0.30^{\circ}$ & $10.13 \pm 0.46^{b c}$ & $9.73 \pm 0.50^{b+1+}$ \\
\hline & Gunsan & $12.65 \pm 0.35^{c * m}$ & $7.70 \pm 0.25^{b}$ & $10.56 \pm 0.16^{c}$ & $11.08 \pm 0.60^{0^{\circ}}$ & $6.51 \pm 0.02^{\mathrm{a}}$ \\
\hline \multirow[t]{2}{*}{ Lysine } & Goheung & $11.60 \pm 0.51^{b}$ & $8.39 \pm 0.09^{\mathrm{a}^{4+*}}$ & $13.17 \pm 0.33^{\mathrm{ctu}}$ & $11.42 \pm 0.57 \mathrm{~b}$ & $10.54 \pm 0.51^{b^{2 *+}}$ \\
\hline & Gunsan & $11.01 \pm 0.31^{\circ}$ & $7.32 \pm 0.23^{\mathrm{a}}$ & $10.66 \pm 0.11^{\circ}$ & $9.91 \pm 0.58^{\mathrm{b}}$ & $6.91 \pm 0.03^{\mathrm{a}}$ \\
\hline \multirow[t]{2}{*}{ Histidine } & Goheung & $3.32 \pm 0.14^{c}$ & $2.42 \pm 0.03^{\mathrm{a}^{+4+t}}$ & $3.28 \pm 0.10^{c^{*}}$ & $3.20 \pm 0.14^{\mathrm{bct}}$ & $2.93 \pm 0.16^{b^{+* t+}}$ \\
\hline & Gunsan & $3.22 \pm 0.09^{d}$ & $2.06 \pm 0.05^{b}$ & $3.11 \pm 0.01^{\mathrm{cd}}$ & $2.93 \pm 0.16^{c}$ & $1.78 \pm 0.12^{\mathrm{a}}$ \\
\hline \multirow[t]{2}{*}{ Valine } & Goheung & $12.17 \pm 0.53^{c^{*}}$ & $8.46 \pm 0.04^{b^{2+*}}$ & $7.90 \pm 0.21^{\mathrm{ab}^{*}}$ & $7.62 \pm 0.33^{a^{*}}$ & $7.20 \pm 0.34^{\mathrm{a}^{3+1+}}$ \\
\hline & Gunsan & $11.33 \pm 0.29^{d}$ & $7.37 \pm 0.25^{\circ}$ & $7.34 \pm 0.11^{\circ}$ & $6.68 \pm 0.31^{b}$ & $5.02 \pm 0.01^{a}$ \\
\hline \multirow[t]{2}{*}{ Arginine } & Goheung & $13.39 \pm 0.58^{\mathrm{a}}$ & $10.17 \pm 0.09^{\mathrm{a}^{\mathrm{at}}}$ & $12.20 \pm 0.34^{c^{*}}$ & $11.79 \pm 0.70^{\mathrm{bc}+*}$ & $10.85 \pm 0.37^{\mathrm{ab}^{\mathrm{a}+*}}$ \\
\hline & Gunsan & $14.17 \pm 0.41^{\circ}$ & $9.05 \pm 0.26^{b}$ & $11.51 \pm 0.17^{\circ}$ & $6.89 \pm 0.01^{\mathrm{a}}$ & $6.90 \pm 0.04^{\mathrm{a}}$ \\
\hline
\end{tabular}

Values are mean of three replication $\pm S D ;{ }^{\text {add }}$ Values with the same superscripts in each row are not significantly different at $p<0.05$; ${ }^{\text {Ns }}$ it is not statistically significant; ${ }^{*} p<0.05,{ }^{* *} p<0.01$ and ${ }^{* * *} p<0.001$, indicating significant difference between Gohueng and Gunsan group 
Table 4 : Comparison of non-essential amino acid content of Pyropia yezoensis (g $\left.100 \mathrm{~g}^{-1} \mathrm{~d} . \mathrm{wt}.\right)$

\begin{tabular}{|c|c|c|c|c|c|c|}
\hline & & Nov & Dec & Jan & Feb & Mar \\
\hline \multirow[t]{2}{*}{ Aspartic acid } & Goheung & $34.62 \pm 1.54^{a}$ & $26.84 \pm 0.27^{b^{*}}$ & $22.13 \pm 0.57^{\mathrm{a}^{\mathrm{at}}}$ & $22.01 \pm 1.01^{\mathrm{a}}$ & $20.56 \pm 1.01^{\mathrm{a}+*+}$ \\
\hline & Gunsan & $37.10 \pm 1.05^{\mathrm{d}^{+}}$ & $25.27 \pm 0.75^{c}$ & $20.62 \pm 0.31^{b}$ & $20.72 \pm 1.12^{b}$ & $13.71 \pm 0.04^{\mathrm{a}}$ \\
\hline \multirow[t]{2}{*}{ Glycine } & Goheung & $26.19 \pm 1.18^{d}$ & $21.69 \pm 0.16^{c^{c t}}$ & $17.11 \pm 0.41^{1^{5+}}$ & $15.54 \pm 0.73^{\mathrm{ab}}$ & $15.18 \pm 0.79^{\mathrm{a} a+1}$ \\
\hline & Gunsan & $25.47 \pm 0.73^{d}$ & $19.26 \pm 0.58^{c}$ & $15.85 \pm 0.28^{b}$ & $15.20 \pm 0.81^{b}$ & $11.18 \pm 0.04^{\mathrm{a}}$ \\
\hline \multirow[t]{2}{*}{ Tyrosine } & Goheung & $3.21 \pm 0.16^{c}$ & $2.81 \pm 0.00^{\mathrm{b}}$ & $1.57 \pm 0.05^{\mathrm{a}}$ & $5.25 \pm 0.22^{\circ}$ & $4.88 \pm 0.26^{\mathrm{d}^{+* t}}$ \\
\hline & Gunsan & $4.81 \pm 0.13^{b^{* * *}}$ & $3.16 \pm 0.11^{\mathrm{a}^{*}}$ & $4.64 \pm 0.08^{b^{* * *}}$ & $5.67 \pm 0.32^{\mathrm{a}}$ & $3.26 \pm 0.01^{\mathrm{a}}$ \\
\hline \multirow[t]{2}{*}{ Serine } & Goheung & $12.21 \pm 0.56^{\mathrm{b}}$ & $9.61 \pm 0.10^{\mathrm{a}^{* *}}$ & $12.15 \pm 0.34^{b^{+}}$ & $11.71 \pm 0.52^{b}$ & $11.35 \pm 0.56^{b+1+1}$ \\
\hline & Gunsan & $12.80 \pm 0.38^{\mathrm{c}}$ & $8.63 \pm 0.23^{\mathrm{a}}$ & $11.54 \pm 0.17^{b}$ & $11.43 \pm 0.58^{b}$ & $7.92 \pm 0.01^{\mathrm{a}}$ \\
\hline \multirow[t]{2}{*}{ Glutamic acid } & Goheung & $37.81 \pm 1.68^{\mathrm{c}}$ & $27.39 \pm 0.27^{b}$ & $23.61 \pm 0.64^{\mathrm{a}}$ & $26.42 \pm 1.21^{\mathrm{b}+4}$ & $22.78 \pm 1.13^{\mathrm{a}^{* *}}$ \\
\hline & Gunsan & $41.48 \pm 1.19^{e^{*}}$ & $27.27 \pm 0.76^{d}$ & $23.49 \pm 0.34^{c}$ & $21.59 \pm 1.19^{b}$ & $15.92 \pm 0.04^{\mathrm{a}}$ \\
\hline \multirow[t]{2}{*}{ Alanine } & Goheung & $23.36 \pm 1.05^{b}$ & $17.65 \pm 0.15^{\mathrm{a}}$ & $31.25 \pm 0.90^{c}$ & $35.54 \pm 1.61^{d}$ & $30.56 \pm 1.56^{c+*+1}$ \\
\hline & Gunsan & $24.66 \pm 0.72^{b}$ & $23.25 \pm 0.64^{b^{t+m}}$ & $34.72 \pm 0.50^{c^{c+}}$ & $35.52 \pm 1.90^{\circ}$ & $20.11 \pm 0.03^{\mathrm{a}}$ \\
\hline \multirow[t]{2}{*}{ Proline } & Goheung & $14.03 \pm 0.57^{\mathrm{b}}$ & $10.60 \pm 0.02^{\mathrm{a}}$ & $11.32 \pm 0.32^{*^{*}}$ & $10.64 \pm 0.48^{\mathrm{a}}$ & $10.40 \pm 0.52^{a^{a+*}}$ \\
\hline & Gunsan & $15.45 \pm 0.07^{\mathrm{d}^{+t}}$ & $10.57 \pm 0.23^{\mathrm{bc}}$ & $10.88 \pm 0.11^{c}$ & $10.17 \pm 0.55^{b}$ & $8.02 \pm 0.06^{\mathrm{a}}$ \\
\hline
\end{tabular}

Values are mean of three replication $\pm S D$; ${ }^{\text {add }}$ Values with the same superscripts in each row are not significantly different at $p<0.05$; ${ }^{\text {Ns }}$ it is not statistically significant; ${ }^{*} p<0.05,{ }^{* *} p<0.01$ and ${ }^{* * *} p<0.001$, indicating significant difference between Gohueng and Gunsan group

Table 5 : Macro mineral contents of Pyropia yezoensis laver (mg $100 \mathrm{~g}^{-1} \mathrm{~d}$. wt.)

\begin{tabular}{lllllll}
\hline Macro-M & & Nov. & Dec. & Jan. & Feb. & Mar. \\
\hline $\mathrm{Ca}$ & Goheung & $136.11 \pm 1.30 \mathrm{a}$ & $183.85 \pm 1.37 \mathrm{~b}$ & $151.54 \pm 12.34 \mathrm{ab}$ & $263.34 \pm 10.61 \mathrm{c}^{* *}$ & $178.56 \pm 30.84 \mathrm{~b}$ \\
& Gunsan & $196.54 \pm 1.41 \mathrm{c}^{* * *}$ & $185.15 \pm 2.63 \mathrm{bc}$ & $153.53 \pm 8.33 \mathrm{a}$ & $226.72 \pm 13.1 \mathrm{~d}$ & $164.74 \pm 15.27 \mathrm{ab}$ \\
$\mathrm{K}$ & Goheung & $2496.81 \pm 17.58 \mathrm{~b}^{* *}$ & $2498.44 \pm 24.43 \mathrm{~b}^{* * *}$ & $2493.53 \pm 278.34 \mathrm{~b}^{*}$ & $1647.87 \pm 33.83 \mathrm{a}$ & $2078.61 \pm 362.37 \mathrm{ab}$ \\
& Gunsan & $1521.63 \pm 9.21 \mathrm{a}$ & $1768.41 \pm 10.50 \mathrm{ab}$ & $1899.31 \pm 88.49 \mathrm{~b}$ & $1879.99 \pm 149.60 \mathrm{~b}^{*}$ & $1929.23 \pm 220.36 \mathrm{~b}$ \\
$\mathrm{Mg}$ & Goheung & $183.33 \pm 0.86 \mathrm{~b}^{* * *}$ & $135.53 \pm 1.28 \mathrm{a}$ & $199.32 \pm 15.76 \mathrm{bc}$ & $146.92 \pm 5.75 \mathrm{a}$ & $227.39 \pm 25.95 \mathrm{c}$ \\
& Gunsan & $103.24 \pm 0.31 \mathrm{a}$ & $162.36 \pm 1.41 \mathrm{~b}^{* * *}$ & $183.25 \pm 12.23 \mathrm{bc}$ & $203.84 \pm 16.67 \mathrm{~cd} \mathrm{~d}^{* *}$ & $232.77 \pm 22.49 \mathrm{~d}$ \\
$\mathrm{Na}$ & Goheung & $644.84 \pm 1.39 \mathrm{~d}^{* * *}$ & $308.72 \pm 2.77 \mathrm{~b}^{* * *}$ & $401.76 \pm 32.01 \mathrm{c}^{*}$ & $142.91 \pm 7.62 \mathrm{a}$ & $454.02 \pm 66.60 \mathrm{c}$ \\
& Gunsan & $79.89 \pm 0.05 \mathrm{a}$ & $291.21 \pm 1.45 \mathrm{~b}$ & $343.79 \pm 19.11 \mathrm{bc}$ & $397.17 \pm 37.67 \mathrm{c}^{* * *}$ & $568.16 \pm 63.43 \mathrm{~d}$ \\
$\mathrm{P}$ & Goheung & $485.06 \pm 2.47 \mathrm{ab}$ & $433.24 \pm 3.60 \mathrm{a}$ & $524.66 \pm 28.13 \mathrm{~b}$ & $481.57 \pm 27.07 \mathrm{ab}$ & $527.62 \pm 28.77 \mathrm{~b}^{* * *}$ \\
& Gunsan & $586.50 \pm 3.16 \mathrm{c}^{* * *}$ & $452.47 \pm 3.99 \mathrm{~b}^{* *}$ & $547.14 \pm 41.04 \mathrm{c}$ & $588.03 \pm 43.30 \mathrm{c}^{*}$ & $242.12 \pm 19.64 \mathrm{a}$ \\
\hline
\end{tabular}

Values are mean of three replication $\pm S D$; ${ }^{\text {a-d }}$ Values with the same superscripts in each row are not significantly different at $p<0.05$; ${ }^{\text {Ns }}$ it is not statistically significant; ${ }^{*} p<0.05,{ }^{* *} p<0.01$ and ${ }^{* * *} p<0.001$, indicating significant difference between Gohueng and Gunsan group

Five macrominerals $(\mathrm{Ca}, \mathrm{K}, \mathrm{Mg}, \mathrm{Na}$ and $\mathrm{P})$ and four microminerals ( $\mathrm{Mn}, \mathrm{Fe}, \mathrm{Cu}$ and $\mathrm{Zn}$ ) were measured in Pyropia samples collected at the Gunsan and Goheung cultivation sites. Mineral content in algae may also be affected by factors such as algal species, oceanic residence time, geographical location of harvest, wave exposure, season and processing method (Yoshie et al., 1994). K was present in highest concentrations, followed by $\mathrm{P}, \mathrm{Na}, \mathrm{Ca}$ and $\mathrm{Mg}$. The concentration of $\mathrm{K}$ in Pyropia cultivated at

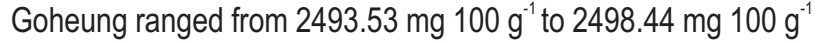
from November to January, which was higher than the concentration in Pyropia cultivated at Gunsan $(P<0.05)$. Similarly, Ruperez (2002) found that $K$ was the most abundant mineral in Chodrus and Pyropia, occurring at concentrations from 3,184 to $3,500 \mathrm{mg} 100 \mathrm{~g}^{-1}$. Macro- and micromineral contents vary by season and region. Conversely, the levels of $\mathrm{Ca}$ and $\mathrm{P}$ were higher in Pyropia samples from Gunsan than in Pyropia samples from Goheung from November to February $(P<0.05)$. $\mathrm{Mg}$ and Na concentrations exhibited irregular patterns over the harvesting period. Among the microminerals, Fe was present at highest

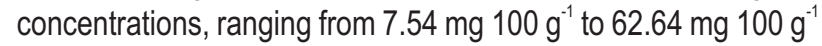
(Table 5). Fe concentration was higher in Pyropia from Gunsan in November, February and March but in Pyropiafrom Goheung in January and February. $\mathrm{Mn}$ and $\mathrm{Cu}$ followed similar pattern as $\mathrm{Fe}$. The $\mathrm{Zn}$ level was higher in Pyropia from Gunsan than in Pyropia from Goheung from November to February (Table 6).

The present study, confirmed that environmental conditions have an impact on biosynthetic pathways. The physical parameters of seawater have a major influence on Pyropia's growth and development, including its carbohydrate, protein, lipid, ash, amino acid and mineral contents. While proximate composition varied temporally and spatially; these effects were not statistically significant. Conversely, amino acid and mineral contents differed significantly depending on both 


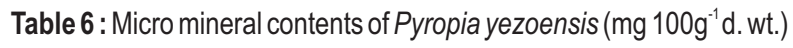

\begin{tabular}{lllllll}
\hline Micro-M & & Nov. & Dec. & Jan. & Feb. & Mar. \\
\hline $\mathrm{Mn}$ & Goheung & $2.42 \pm 0.01 \mathrm{c}$ & $2.22 \pm 0.03 \mathrm{c}^{* * *}$ & $2.19 \pm 0.09 \mathrm{c}^{* *}$ & $1.32 \pm 0.09 \mathrm{a}$ & $1.71 \pm 0.18 \mathrm{~b}$ \\
& Gunsan & $3.29 \pm 0.02 \mathrm{c}^{* * *}$ & $1.62 \pm 0.02 \mathrm{a}$ & $1.63 \pm 0.14 \mathrm{a}$ & $1.97 \pm 0.16 \mathrm{~b}^{* *}$ & $1.94 \pm 0.14 \mathrm{~b}$ \\
$\mathrm{Fe}$ & Goheung & $12.09 \pm 0.05 \mathrm{~b}$ & $12.67 \pm 0.09 \mathrm{~b}^{* * *}$ & $24.68 \pm 1.21 \mathrm{~d}^{* * *}$ & $7.96 \pm 0.43 \mathrm{a}$ & $14.50 \pm 0.62 \mathrm{c}$ \\
$\mathrm{Cu}$ & Gunsan & $14.64 \pm 0.01 \mathrm{ab} \mathrm{b}^{* * *}$ & $9.15 \pm 0.1 \mathrm{a}$ & $7.54 \pm 1.16 \mathrm{a}$ & $26.05 \pm 6.62 \mathrm{~b}^{* *}$ & $62.64 \pm 11.05 \mathrm{c}^{* *}$ \\
& Goheung & $0.56 \pm 0.01 \mathrm{~b}$ & $0.96 \pm 0.02 \mathrm{c}^{* * *}$ & $0.35 \pm 0.09 \mathrm{a}$ & $0.51 \pm 0.06 \mathrm{~b}$ & $0.27 \pm 0.06 \mathrm{a}$ \\
$\mathrm{Zn}$ & Gunsan & $0.75 \pm 0.01 \mathrm{c}^{* * *}$ & $0.47 \pm 0.01 \mathrm{~b}$ & $0.24 \pm 0.01 \mathrm{a}$ & $0.76 \pm 0.04 \mathrm{c}^{* *}$ & $0.28 \pm 0.06 \mathrm{a}$ \\
& Goheung & $3.54 \pm 0.03 \mathrm{NS}$ & $3.09 \pm 0.01$ & $3.29 \pm 0.23$ & $3.13 \pm 0.19$ & $3.46 \pm 0.27$ \\
& Gunsan & $8.81 \pm 0.03 \mathrm{c}^{* * *}$ & $3.20 \pm 0.02 \mathrm{ab} \mathrm{b}^{* *}$ & $3.76 \pm 0.42 \mathrm{~b}$ & $3.45 \pm 0.31 \mathrm{ab}$ & $3.08 \pm 0.22 \mathrm{a}$ \\
\hline
\end{tabular}

Values are mean of three replication \pm SD; a-d Values with the same superscripts in each row are not significantly different at $p<0.05$; NS it is not statistically significant; ** $p<0.01$ and ${ }^{* * *} p<0.001$, indicating significant difference between Gohueng and Gunsan group

season and region.

Edible marine seaweeds may be an important source of nutrition, because some of their constituents are nearly or entirely absent from terrestrial vegetation. Based on the results of this study, temporal and spatial variations may influence the proximate composition, amino acid and mineral content of red algae, and knowledge of these patterns may be useful for both producers and consumers.

\section{Acknowledgments}

This research was supported by the Golden Seed Project, Ministry of Agriculture, Food and Rural Affairs (MAFRA), Ministry of Oceans and Fisheries (MOF), Rural Development Administration (RDA), and Korea Forest Service (KFS). It was also partially supported by the Soonchunhyang University.

\section{References}

AOAC. Official methods of analysis. Washington, DC: Association of Official Analytical Chemists (1995).

Cian, R.E., M.A. Fajardo, M. Alaiz, J. Vioque, R.J. González and S.R. Drago: Chemical composition, nutritional and antioxidant properties of the red edible seaweed Porphyra columbina. Int. J. Food Sci. Nutr., 65, 299-305 (2014).

Dawczynski, C., R. Schubert and G. Jahreis: Amino acids, fatty acids, and dietary fibre in edible seaweed products. Food Chem., 103, 891-899 (2007).

Fleurence, J.: Seaweed proteins: Biochemical, nutritional aspects and potential uses. Trends Food Sci. Tech.,10, 25-28 (1999).

Galland-Irmouli, A.V., J. Fleurence, R. Lamghari, M. Luçon, C. Rouxel, O. Barbaroux and J.L. Guéant: Nutritional value of proteins from edible seaweed Palmaria palmata (Dulse). J. Nutr. Biochem., 10, 353-359 (1999).

Gorman, L., G.P. Kraemer, C. Yarish, S.M. Boo and J.K. Kim: The effects of temperature on the growth and nitrogen content of Gracilaria vermiculophylla and Gracilaria tikvahiae from LIS, USA. Algae, 32 , 57-66 (2017).

Hwang, E.S., K.N. Ki and H.Y. Chung: Proximate composition, amino acid, mineral and heavy metal content of dried Laver. Prev. Nutr. Food Sci.,18, 139-144 (2013).
Jung, S.M., S.G. Kang, H.J. Lee, J.S. Son, J.H. Jeon and H.W. Shin: Proximate composition and mineral content, amino acid of laver based on culture areas. Korean J. Environ. Ecol., 30, 98-103 (2016a).

Jung, S.M., S.G., Kang, J.S. Son, J.H. Jeon, H.J. Lee and H.W. Shin: Temporal and spatial variations in the proximate composition, amino acid and mineral content of Pyropiaye zoensis. J. Appl. Phycol., 28, 3459-3467 (2016b).

Jurkovic, N., N. Kolb and I. Colic: Nutritive value of marine algae Laminaria japonica and Undaria pinnatifida. Die Nahung, 39, 63-66 (1995).

Kishi, K., G. Inone, A. Yoshida, H. Fuwa, H. Koishi, G. Koide, T. Miyoshi, T. Inone, M. Yoshida and A. Omori: Digestibility and energy availability of sea vegetables and fungi in man. Nutr. Rep. Int., 26, 183-192 (1982).

Kim, G.H., K.H. Moon, J.Y. Kim, J. Shim and T.A. Klochkova: A revaluation of algal diseases in Korean Pyropia (Porphyra) sea farms and their economic impact. Algae, 29, 249 (2014).

Lahaye, M. and B. Kaeffer: Seaweed dietary fibres: Structure, physicochemical and biological properties relevant to intestinal physiology. Sci. Aliments, 17, 619-639 (1997).

Lee, H.J., J.I. Choi and S.J. Choi: Physiological activities and amino acid compositions of Korean dried laver Porphyra products. Korean $\mathrm{J}$. FishAquat. Sci., 45, 409-413 (2012).

Lobban, C.S., P.J. Harrison and M.J. Duncan: Physiological ecology of seaweeds. Cambridge University Press.Cambridge, p. 242 (1985).

Mabeau, S., E. Cavaloc, J. Fleurence and M. Lahaye: New seaweed based ingredients for the food industry. Int. Food Ing., 3, 38-45 (1992).

Marinho-Soriano, E., P.C. Fonseca, M.A.A. Carneiro and W.S.C. Moreira: Seasonal variation in the chemical composition of two tropical seaweeds. Bioresource Technol., 97, 2402-2406 (2006).

Munda, I.M.: Differences in amino acid composition of estuarine and marine fucoids. Aquat. Bot., 3, 273-280 (1977).

Nisizawa, K., H. Noda, R. Kikuchi and T. Watanabe: The main seaweed foods in Japan. In: Twelfth International Seaweed Symposium. Springer Netherlands, pp. 5-29 (1987).

Noda, H.: Biochemical studies on marine algae 2. Relation between quality and chemical composition of Asakusanori. Bull Jpn. Soc. Sci. Fish, 37, 30-34 (1971a).

Noda, H.: Biochemical studies on marine algae. 3. Relation between quality and inorganic constituents of Asakusanori. Bull. Jpn. Soc. Sci. Fish, 37, 35 (1971b). 
Noda, $\mathrm{H}$.: Health benefits and nutritional properties of nori. J. Appl. Phycol., 5, 255-258(1993).

Norziah, M.H. and C.Y. Ching: Nutritional composition of edible seaweed Gracilariachanggi. Food Chem.,68, 69-76 (2000).

Ortega-Calvo, J.J., C. Mazuelos, B. Hermosin and C. Sáiz-Jiménez: Chemical composition of Spirulina and eukaryotic algae food products marketed in Spain. J. Appl. Phycol., 5, 425-435 (1993).

Ortiz, J., N. Romero, P. Robert, J. Araya, J. Lopez-Hernández, C. Bozzo and A. Rios: Dietary fiber, amino acid, fatty acid and tocopherol contents of the edible seaweeds Ulva lactuca and Durvillaea antarctica. Food Chem., 99, 98-104 (2006).

Park, C.K., C.H. Park and J.N. Park: Extractive nitrogenous constituents and their monthly variation of fresh laver Porpyra yezoensis. Food Sci. Biotechnol., 10, 364-374 (2001).

Pérez, A.A., S.S. Farías, A.M. Strobl, L.B. Pérez, C.M. López, A. Piñeiro, R. Otmaro and M.A. Fajardo: Levels of essential and toxic elements in Porphyra columbina and Ulva sp. from San Jorge Gulf, PatagoniaArgentina. Sci. Total Environ., 376, 51-59 (2007).
Phillips, J.A.: Life history studies of Ulva rigida C. Ag. and Ulva stenophylla S. et G. (Ulvaceae, Chlorophyta) in Southern Australia. Bot. Marin., 33, 79-84 (1990).

Rupérez, P.: Mineral content of edible marine seaweeds. Food Chem.,79, 23-26 (2002).

Shin, J.A.: Inheritance mode of some characters of Porphyra yezoensis (Bangiales, Rhodophyta) I. Crispness and free amino acid content in two recombinant wild-types bred by reciprocal crosses. Algae, 12,313-318 (1997).

Shin, D.M., S.R. An, S.K. In and J.G. Koo: Seasonal variation in the dietary fiber, amino acid and fatty acid contents of Porphyra yezoensis. Kor. J. FishAquat. Sci., 46, 337-342 (2013).

Taboada, M.C., R. Millán and M.I. Miguez: Nutritional value of the marine algae wakame (Undaria pinnatifida) and nori (Porphyra purpurea) as food supplements. J. Appl. Phycol., 25, 1271-1276 (2013).

Yoshie, Y., T. Suzuki, T. Shirai and T. Hirano: Dietary fiber and minerals in dried nori of various culture location and prices. Bull. Japan Soc. Scic. Fish, 59, 1763-1768 (1993). 